\title{
Optical Waveguides with Compound Multiperiodic Grating Nanostructures for Refractive Index Sensing
}

\author{
Lars Thorben Neustock, ${ }^{1}$ Sabrina Jahns, ${ }^{1}$ Jost Adam, ${ }^{2}$ and Martina Gerken ${ }^{1}$ \\ ${ }^{1}$ Institute of Electrical Engineering and Information Technology, Christian-Albrechts-Universität zu Kiel, Kaiserstraße 2, \\ 24143 Kiel, Germany \\ ${ }^{2}$ The Mads Clausen Institute, University of Southern Denmark, Alsion 2, 6400 Sønderborg, Denmark
}

Correspondence should be addressed to Martina Gerken; mge@tf.uni-kiel.de

Received 6 August 2015; Accepted 13 October 2015

Academic Editor: Wentao Zhang

Copyright (C) 2016 Lars Thorben Neustock et al. This is an open access article distributed under the Creative Commons Attribution License, which permits unrestricted use, distribution, and reproduction in any medium, provided the original work is properly cited.

\begin{abstract}
The spectral characteristics and refractive index sensitivity of compound multiperiodic grating waveguides are investigated in theory and experiment. Compound gratings are formed by superposition of two or more monoperiodic gratings. Compared to monoperiodic photonic crystal waveguides, compound grating waveguides offer more degrees of design freedom by choice of component grating periods and duty cycles. Refractive index sensing is achieved by evaluating the wavelength or intensity of guided mode resonances in the reflection spectrum. We designed, fabricated, and characterized 24 different compound multiperiodic nanostructured waveguides for refractive index sensing. Simulations are carried out with the Rigorous Coupled Wave Algorithm (RCWA). The resulting spectra, resonance sensitivities, and quality factors are compared to monoperiodic as well as to three selected aperiodic nanostructures (Rudin-Shapiro, Fibonacci, and Thue-Morse). The refractive index sensitivity of the TE resonances is similar for all types of investigated nanostructures. For the TM resonances the compound multiperiodic nanostructures exhibit higher sensitivity values compared to the monoperiodic nanostructure and similar values as the aperiodic nanostructures. No significant influence of the compound grating duty cycles on the sensitivity is observed.
\end{abstract}

\section{Introduction}

Dielectric, periodic photonic nanostructured waveguides are commonly used as transducers for label-free biosensors [1-4]. These systems are based on sampling of molecular binding events with evanescent fields of quasi-guided modes. Changes in resonance wavelength, intensity, or quality factor of quasi-guided modes are monitored by transmission or reflection measurements. Specificity of these sensors is obtained by biofunctionalization of the surface for specific capture. Detection of biomarkers without adding labels, such as fluorescence labels, prevents measurement errors due to labeling and simpler microfluidic test chips are within reach. These sensors belong more generally to the class of optical refractive index (RI) sensors based on monitoring optical resonances, which also encompasses surface plasmon resonance (SPR) sensors and microcavity resonators [5, 6]. For highperformance sensors a high RI sensitivity $S=\Delta \lambda / \Delta n(\Delta \lambda$ : mode shift in wavelength; $\Delta n$ : refractive index change) and a high quality factor $Q=\omega_{r} / \Delta \omega,\left(\omega_{r}\right.$ : resonant frequency; $\Delta \omega$ : resonance width at half maximum) of the mode are necessary [5]. With perturbation theory the wavelength shift $\Delta \lambda$ and correspondingly the frequency shift $\Delta \omega$ of the guided mode resonance are related to the local change in permittivity $\Delta \varepsilon(\vec{r})$ and the local electric field $E(\vec{r})$ at position $\vec{r}$ as given in (1) $[6,7]$ :

$$
\frac{\Delta \lambda}{\lambda}=-\frac{\Delta \omega}{\omega}=\frac{1}{2} \frac{\int d^{3} r \cdot \Delta \varepsilon(\vec{r})|E(\vec{r})|^{2}}{\int d^{3} r \cdot \varepsilon(\vec{r})|E(\vec{r})|^{2}}
$$

The Q-factor correlates with the scattering length and thus the photon lifetime in the nanostructure [8]. This indicates that compound multiperiodic nanostructures scatter photons more efficiently. Ding and Magnusson [9] have demonstrated that the modulation strength defined by the refractive index 


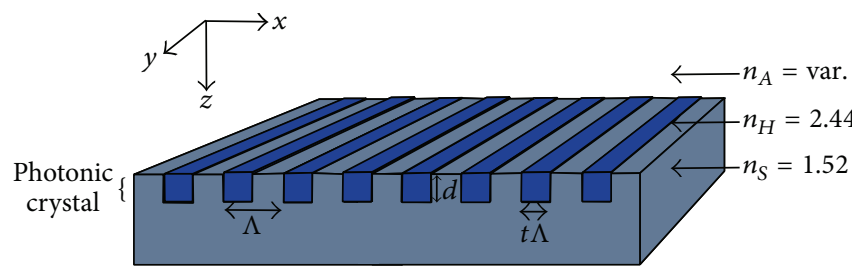

(a) Monoperiodic nanostructure (M)

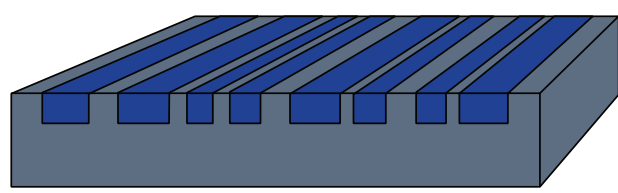

(b) Compound multiperiodic nanostructure (C)

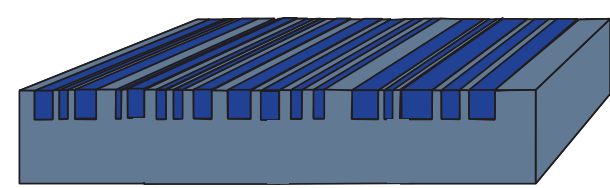

(c) Rudin-Shapiro nanostructure (RS)



(d) Thue-Morse nanostructure (ThM)

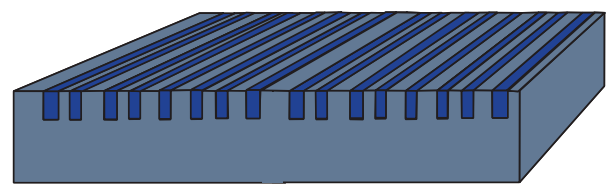

(e) Fibonacci nanostructure (F)

FIGURE 1: Schematics of the investigated types of nanostructured optical waveguides for refractive index sensing. A change in the analyte refractive index $n_{A}$ in the upper hemisphere is detected by changes in the normal-incidence transmission or reflection spectrum. (a) Monoperiodic nanostructure. (b) Compound multiperiodic nanostructure with two periods. (c) Rudin-Shapiro nanostructure. (d) ThueMorse nanostructure. (e) Fibonacci nanostructure.

contrast and the grating depth allows for a tailoring of the Qfactor.

SPR sensors typically offer a good RI sensitivity $S$ but exhibit limited mode quality factors $Q$ due to the metal absorption losses. Purely, dielectric resonator structures, on the other hand, allow for high mode quality factors $Q$. Improved performance of photonic crystal slab sensors has been demonstrated by Nicolaou et al. [10] by employing a photonic crystal with alternating nanohole sizes. Recently, the use of deterministic aperiodic nanostructures for biosensing has been suggested [11-13]. Deterministic aperiodic nanostructures are engineered ordered nanostructures without periodicity [12-16]. The additional degrees of freedom allow for a tailoring of the optical properties. In particular it has been demonstrated that quasi-localized critical modes in aperiodic nanostructures simultaneously exhibit high quality factors $Q$ and high sensitivity $S$ to refractive index changes [11]. For example, a significantly increased RI sensitivity was calculated for Rudin-Shapiro nanostructures compared to band-edge and point-defect modes of periodic photonic crystal nanostructures [12]. Here, we propose the use of compound multiperiodic gratings for refractive index sensing.

Compound gratings are obtained by performing a logical disjunction operation of two or more monoperiodic gratings. Such compound gratings allow for the design of the number, wavelengths, and relative intensities of resonance peaks in the transmission and reflection spectra $[17,18]$. We investigate the spectral characteristics and RI sensitivity of compound multiperiodic gratings in theory and experiment. In a theoretical investigation the performance of multiperiodic grating waveguides is compared to monoperiodic and three selected aperiodic (Rudin-Shapiro, Fibonacci, and
Thue-Morse) nanostructured waveguide gratings. In experiment the characteristics of 24 compound multiperiodic gratings with two and three grating components and different combinations of duty cycles are investigated. A monoperiodic grating is characterized as a reference.

\section{Material and Methods}

2.1. Nanostructures. Five different types of nanostructured waveguide gratings are investigated: monoperiodic (M), compound multiperiodic (C), Rudin-Shapiro (RS), ThueMorse (ThM), and Fibonacci (F) nanostructures. For all structures a substrate refractive index of $n_{S}=1.52$ and a waveguide with refractive index $n_{H}=2.44$ and height $d=100 \mathrm{~nm}$ are assumed. Schematics of the different types of nanostructured waveguides are presented in Figure 1 and Table 1 gives a parameter overview. The upper hemisphere is assumed to be filled with an analyte of refractive index $n_{A}$.

The compound multiperiodic gratings are created by a logical disjunction of two or more monoperiodic gratings with different grating periods $\Lambda_{i}$ and duty cycles $t_{i}$ resulting in compound binary gratings [17]. The aperiodic gratings are obtained by substitution sequences with two letter alphabets $[11,19,20]$. The binary sequences are transformed into onedimensional symmetric nanostructures. For this, we transformed each single sequence component into a structure with $h \mathrm{~nm}$ in $x$-direction and used $\mathrm{A}$ as a representative of high index material and $\mathrm{B}$ as substrate material. Thus, a subsequence of $\mathrm{BBB}$ results in a $3 \cdot h$ long substrate region in $x$ direction. A symmetric refractive index profile is generated by mirroring around $x=0$. 
TABLE 1: Investigated nanostructures. For all structures substrate refractive index $n_{S}=1.52$ and waveguide refractive index $n_{H}=2.44$. $\Lambda$ is the periodicity and $t$ the duty cycle (ratio of high-index material to substrate material in one period). For the aperiodic sequences $h$ is the bit length.

\begin{tabular}{|c|c|c|}
\hline & Type & Description \\
\hline $\mathrm{M}$ & Monoperiodic & $\Lambda=400 \mathrm{~nm} ; t=0.5$ \\
\hline $\mathrm{C} 2$ & $\begin{array}{l}\text { Compound multiperiodic } \\
\text { nanostructure with two } \\
\text { periods }\end{array}$ & $\begin{array}{l}\Lambda_{1}=350 \mathrm{~nm} ; \Lambda_{2}=450 \mathrm{~nm} ; \Lambda=3150 \mathrm{~nm} \\
\text { Duty cycle combinations: } \\
t_{1}=0.14\left|t_{2}=0.5 ; t_{1}=0.29\right| t_{2}=0.5 ; t_{1}=0.5 \mid t_{2}=0.5 ; \\
t_{1}=0.57\left|t_{2}=0.5 ; t_{1}=0.14\right| t_{2}=0.22 ; t_{1}=0.29 \mid t_{2}=0.22 \\
t_{1}=0.42\left|t_{2}=0.22 ; t_{1}=0.57\right| t_{2}=0.22\end{array}$ \\
\hline $\mathrm{C} 3 \mathrm{a}$ & $\begin{array}{l}\text { Compound multiperiodic } \\
\text { nanostructure with three } \\
\text { periods }\end{array}$ & $\begin{array}{l}\Lambda_{1}=350 \mathrm{~nm} ; \Lambda_{2}=400 \mathrm{~nm} ; \Lambda_{3}=450 \mathrm{~nm}: \Lambda=25200 \mathrm{~nm} \\
\text { Duty cycle combinations: } \\
t_{1}=0.14\left|t_{2}=0.25\right| t_{3}=0.33 ; t_{1}=0.29\left|t_{2}=0.25\right| t_{3}=0.33 ; \\
t_{1}=0.42\left|t_{2}=0.25\right| t_{3}=0.33 ; t_{1}=0.57\left|t_{2}=0.25\right| t_{3}=0.33 ; \\
t_{1}=0.14\left|t_{2}=0.25\right| t_{3}=0.22: t_{1}=0.29\left|t_{2}=0.25\right| t_{3}=0.22 ; \\
t_{1}=0.42\left|t_{2}=0.25\right| t_{3}=0.22 ; t_{1}=0.57\left|t_{2}=0.25\right| t_{3}=0.22\end{array}$ \\
\hline $\mathrm{C} 3 \mathrm{~b}$ & $\begin{array}{l}\text { Compound multiperiodic } \\
\text { nanostructure with three } \\
\text { periods }\end{array}$ & $\begin{array}{l}\Lambda_{1}=300 \mathrm{~nm} ; \Lambda_{2}=400 \mathrm{~nm} ; \Lambda_{3}=500 \mathrm{~nm}: \Lambda=6000 \mathrm{~nm} \\
\text { Duty cycle combinations: } \\
t_{1}=0.14\left|t_{2}=0.25\right| t_{3}=0.3 ; t_{1}=0.33\left|t_{2}=0.25\right| t_{3}=0.3 ; \\
t_{1}=0.5\left|t_{2}=0.25\right| t_{3}=0.3 ; t_{1}=0.66\left|t_{2}=0.25\right| t_{3}=0.3 ; \\
t_{1}=0.14\left|t_{2}=0.25\right| t_{3}=0.2: t_{1}=0.33\left|t_{2}=0.25\right| t_{3}=0.2 ; \\
t_{1}=0.5\left|t_{2}=0.25\right| t_{3}=0.2 ; t_{1}=0.66\left|t_{2}=0.25\right| t_{3}=0.2\end{array}$ \\
\hline $\mathrm{C} 4$ & $\begin{array}{l}\text { Compound multiperiodic } \\
\text { nanostructure with four } \\
\text { periods }\end{array}$ & $\Lambda_{1}=250 \mathrm{~nm} ; \Lambda_{2}=350 \mathrm{~nm} ; \Lambda_{3}=400 \mathrm{~nm} ; \Lambda_{4}=450 \mathrm{~nm}: \Lambda=126000 \mathrm{~nm} ; t_{1-4}=0.2$ \\
\hline RS & Rudin-Shapiro & $\begin{array}{l}\text { Substitution } \mathrm{AA} \rightarrow \mathrm{AAAB}, \mathrm{AB} \rightarrow \mathrm{AABA}, \mathrm{BA} \rightarrow \mathrm{BBAB}, \mathrm{BB} \rightarrow \mathrm{BBBA} ; h=25 \mathrm{~nm} ; \\
N=128 \text { series components; } \\
\Lambda=6375 \mathrm{~nm} \text { supercell periodicity }\end{array}$ \\
\hline ThM & Thue-Morse & $\begin{array}{l}\text { Substitution } \mathrm{A} \rightarrow \mathrm{AB}, \mathrm{B} \rightarrow \mathrm{BA} ; h=50 \mathrm{~nm} ; N=128 \text { series components; } \Lambda= \\
12750 \mathrm{~nm} \text { supercell periodicity }\end{array}$ \\
\hline $\mathrm{F}$ & Fibonacci & $\begin{array}{l}\text { Substitution } \mathrm{A} \rightarrow \mathrm{AB}, \mathrm{B} \rightarrow \mathrm{A} ; h=50 \mathrm{~nm} ; N=144 \text { series components; } \Lambda= \\
14350 \mathrm{~nm} \text { supercell periodicity }\end{array}$ \\
\hline
\end{tabular}

2.2. Simulation Methods. We implemented a Rigorous Coupled Wave Algorithm (RCWA), also known as Fourier modal method, following Moharam et al. [21]. All simulations were carried out in MATLAB. The Fourier components of the monoperiodic nanostructure $\mathrm{M}$ were calculated according to [21] using 16 Fourier components. The number of components required for an accurate result was derived by a convergence analysis with different component numbers. As stated in [22] the first nonzero Fourier component is connected with coupling waves into (or out off) the TE mode. Thus, a higher number of components are unnecessary. The TM mode behaves accordingly.

We calculated the Fourier components for the multiperiodic gratings by recreating the logical disjunction in the spectrum by (1) the addition of the two input monoperiodic spectra (padded with zeros), (2) the subtraction of the convolution of both, and (3) new calculation of the central component [17]. Convergence analyses yielded the necessary number of Fourier components (adjusted for fast Fourier transform) as 64 components for two-periodic, 512 components for threeperiodic, and 1024 components for four-periodic compound gratings. Again, the first component of the superimposed monoperiodic Fourier spectra is essential for the wave coupling into a (quasi-) guided mode. However, due to the disjunction, these components had higher indices in the resulting spectra [17]. Thus, higher numbers of components are necessary for more complex multiperiodic structures.

To implement aperiodic structures into our simulation, we chose a supercell approach $[23,24]$. Using a supercell means, to implement a nanostructure with such a large periodicity, that the calculated results should not differ from a real aperiodic structure in a substantial way. We tried different supercell lengths and decided to transform $N=128$ series components for the RS and ThM structures and $N=$ 144 for the Fibonacci sequence. To obtain the spectrum of the aperiodic gratings, we calculated the Fourier components of a monoperiodic structure with just one high index region and a period as high as the supercell length and positioned this structure via a modulation of the Fourier spectrum. We repeated this process iteratively until the whole sequence was transformed. Due to the complex nature of the resulting spectrum, 1500 components were used for calculation of the results.

For calculation of the RI sensitivity $S=\Delta \lambda / \Delta n$ the reflection spectra for analyte refractive indices of $n_{A}=1$ (air) and $n_{A}=1.33$ (water) are simulated. $\Delta \lambda$ is obtained as the shift of the peak wavelength of the considered resonance. The quality factor $Q$ is calculated for the simulated results by fitting a Fano function and for the experimental results by fitting a Lorentz function to the resonance peak. 


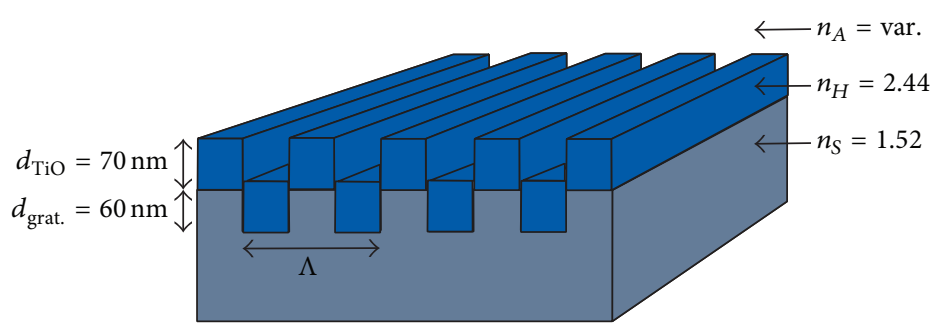

(a)

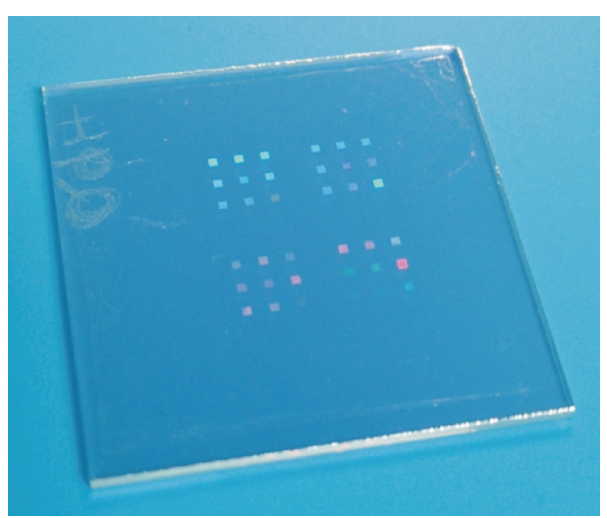

(b)



(c)

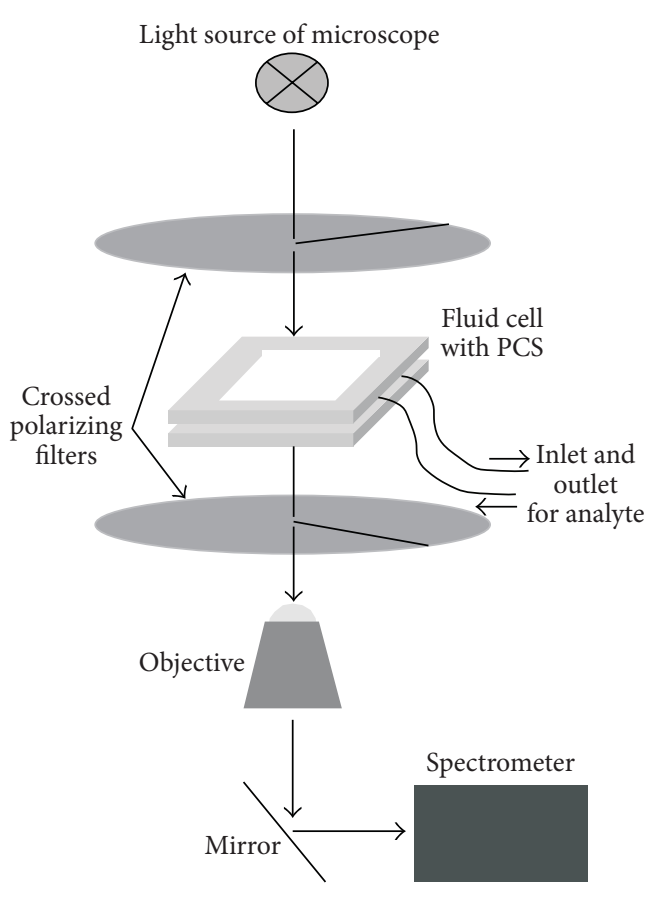

(d)

Figure 2: (a) Schematic of fabricated samples. (b) Photograph of fabricated sample. (c) Photograph (stitched) of grating fields observed with experimental setup with crossed polarization filters (camera at output instead of spectrometer). (d) Schematic of spectrometer setup for optical characterization of nanostructured waveguides.

2.3. Experimental Methods. Nanostructured waveguides were fabricated by ultraviolet (UV) nanoimprint lithography and subsequent evaporation of titania $\left(\mathrm{TiO}_{2}\right)$ [25]. First, an electron-beam written nickel master with $500 \times 500 \mu \mathrm{m}^{2}$ large nanostructured fields is replicated into a polydimethylsiloxane (PDMS, Sylgard 184, and Curing Agent by DOW Corning) stamp. Second, Amoprime and the nanoimprint resist Amonil (both $A M O \mathrm{GmbH}$ ) are spin coated onto glass substrates, the PDMS stamp is placed on top, and the resist is hardened with a UV halogen lamp. After pulling off the PDMS stamp, a $70 \mathrm{~nm}$ thick $\mathrm{TiO}_{2}$ layer is deposited by reactive sputtering in the final third step. A photograph of the resulting sample is shown in Figure 2(b). The nanostructured fields are visible as colored shining rectangles because of light diffraction on the linear gratings.
For characterization of the different nanostructured fields for refractive index sensing, the sample is placed within a fluid cell. An O-ring is placed between the $25 \times 25 \mathrm{~mm}^{2}$ large sample and a glass substrate. For analyte exchange two butterfly cannulas are pierced through the O-ring and two substrate holders with screws keep the assembly in place. As depicted in Figure 2(d) the fluid cell is placed on a microscope table between two crossed linear polarization filters. The crossed polarization filters suppress background light. Only light interacting with the nanostructured waveguide passes the second polarization filter [26]. The halogen lamp of the microscope is used as a broadband excitation source. Via an aperture before the halogen lamp the excitation light is restricted to a single nanostructured field. The transmitted light is guided through the microscope objective via a mirror 




(a) $\mathrm{M}: \Lambda=400 \mathrm{~nm} ; t=0.5(\mathrm{sim}$.)



(b) $\mathrm{M}: \Lambda=400 \mathrm{~nm} ; t=0.5$ (meas.)

FiguRE 3: (a) Simulated and (b) measured reflectance spectra of the monoperiodic nanostructure $\mathrm{M}(\Lambda=400 \mathrm{~nm}, t=0.5)$. Fano-like peaks are observed for the simulation, while the measured spectra exhibit Lorentzian peaks due to the measurement with crossed polarization filters for background suppression.

to the spectrometer. The fluid cell is filled sequentially with air and water. The large refractive index change allows for an easily visible change in the spectrum.

\section{Results and Discussion}

The results are sorted by nanostructure type. In Section 3.1 the simulation and experimental results for a monoperiodic nanostructure are presented, in Section 3.2 the simulation and experimental results for compound multiperiodic nanostructures are presented, and in Section 3.3 the simulation results for aperiodic nanostructures are presented.

3.1. Monoperiodic Nanostructures. The simulated and measured spectra for the monoperiodic nanostructure $M$ with period $\Lambda=400 \mathrm{~nm}$ and a duty cycle of $t=0.5$ are presented in Figure 3. Both spectra show the expected resonance behaviour in the visible light regime. For the simulation the typical Fano resonance line shape is observed [27]. The measured spectra exhibit Lorentzian peaks due to the measurement with crossed polarization filters for background suppression [26]. Agreement is observed for the peak positions for TE polarization between measurement and simulation. The different quality factors may be attributed to the simplified simulation structure compared to the experimental nanostructure that has a high refractive index layer covering the complete nanostructured surface. Nevertheless, good agreement is obtained for the sensitivities. In simulation a sensitivity of $S=33 \mathrm{~nm} / \mathrm{RIU}$ is calculated for the TE resonance and $19 \mathrm{~nm} / \mathrm{RIU}$ for the TM resonance. Experimentally a sensitivity of $S=32 \mathrm{~nm} / \mathrm{RIU}$ is measured for the TE resonance.

3.2. Compound Multiperiodic Nanostructures. 24 different compound multiperiodic gratings $(\mathrm{C} 2, \mathrm{C} 3 \mathrm{a}$, and $\mathrm{C} 3 \mathrm{~b}$ with eight different duty cycle combinations each) were fabricated and characterized experimentally. Figures 4-7 show the simulated and measured reflection spectra for selected compound multiperiodic gratings. As expected from the superposition process the spectra for multiperiodic nanostructures show dominant peaks for each superimposed periodicity $[17,18]$. Additionally, the simulated spectra show small resonances due to the convolution part in the Fourier spectra, which creates additional nonzero Fourier components.

The dominant resonance peaks show in the simulation a lower intensity than in the monoperiodic case. The resulting resonance peak was at $100 \%$ for the monoperiodic case, as also expected from $[22,28]$. The peak intensity decreases as more grating components are included in the compound grating. Accordingly, there is a tradeoff between peak intensity and number of modes (and thus resonance peaks) supported by the photonic crystal waveguide. However, as also shown in [22], the coupling factor of the modes depends on the duty cycle of the photonic crystal. The duty cycle strongly influences the first Fourier component. Figure 8 presents measured spectra for compound multiperiodic structures with the same underlying periodicities but different duty cycles. The relative intensity of the resonance peaks may be tailored by adjusting the duty cycles.

Table 2 summarizes the sensitivities calculated from the spectral resonance shift between air and water as analytes. In the experiments and simulations no significant influence of the grating duty cycles on the sensitivity was observed. Thus, Table 2 gives the averaged sensitivity for the eight different duty cycle combinations for each grating type and the standard deviation for the measurement results. The simulation results correspond to the duty cycles given in Figures 47. Overall, good agreement is observed between measured values and simulation results.

The sensitivities of corresponding TE resonances appear independent of the number of components in the compound grating. On the other hand, for the TM resonances the sensitivity increases for more grating components. This observation is similar to observations made for aperiodic twodimensional photonic crystals $[11,29]$. The highest sensitivity 


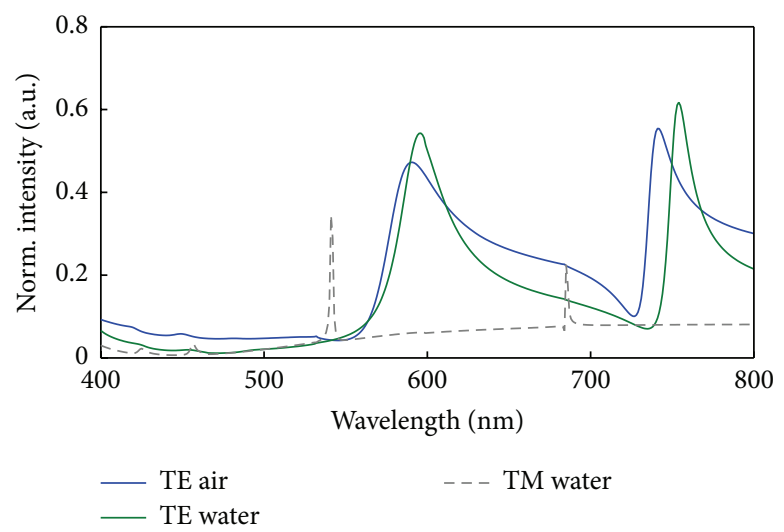

(a) $\mathrm{C} 2: t_{1}=0.29 \mid t_{2}=0.5(\operatorname{sim}$.)



(b) $\mathrm{C} 2: t_{1}=0.29 \mid t_{2}=0.5$ (meas.)

Figure 4: (a) Simulated and (b) measured reflectance spectra of the compound multiperiodic nanostructure C2 $\left(t_{1}=0.29 \mid t_{2}=0.5\right)$. The second dominant peak is not in the experimental range.

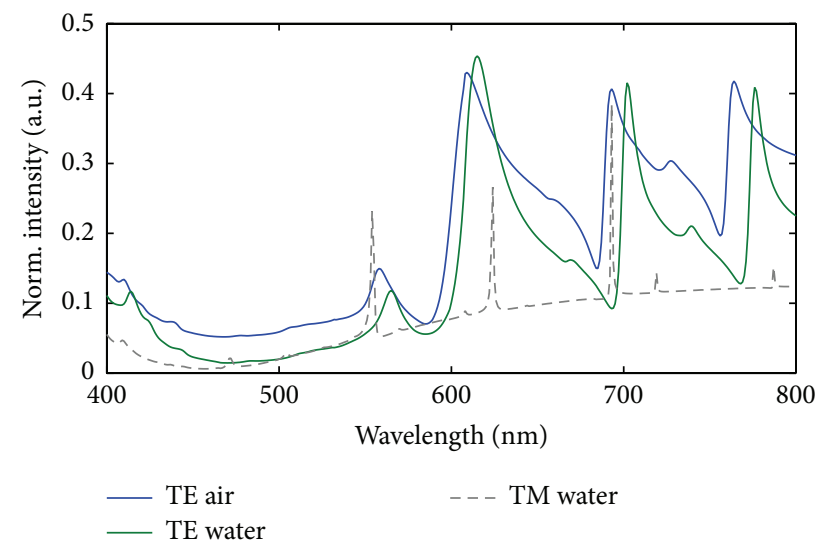

(a) C3a: $t_{1}=0.29\left|t_{2}=0.25\right| t_{3}=0.33$ (sim.)

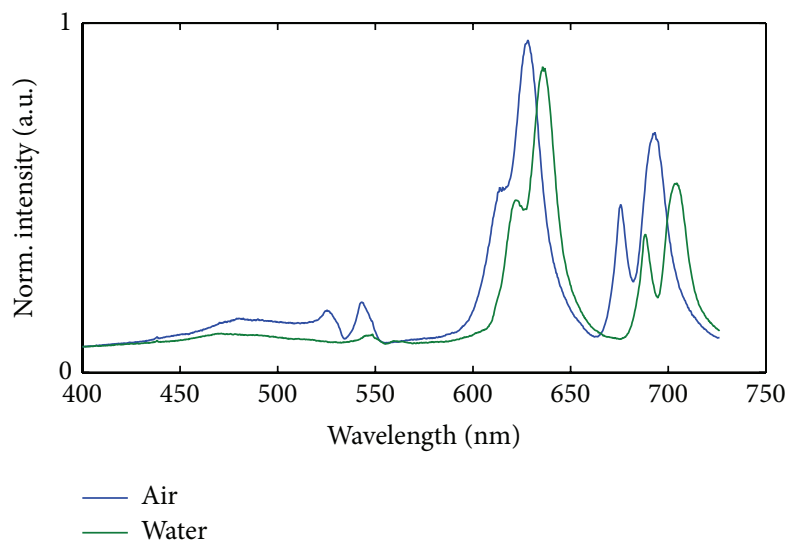

(b) C3a: $t_{1}=0.29\left|t_{2}=0.25\right| t_{3}=0.33$ (meas.)

FIGURE 5: (a) Simulated and (b) measured reflectance spectra of the compound multiperiodic nanostructure C3a $\left(t_{1}=0.29\left|t_{2}=0.25\right| t_{3}=\right.$ $0.33)$. The third dominant peak is not in the experimental range.



(a) C3b: $t_{1}=0.5\left|t_{2}=0.25\right| t_{3}=0.2(\operatorname{sim}$.)

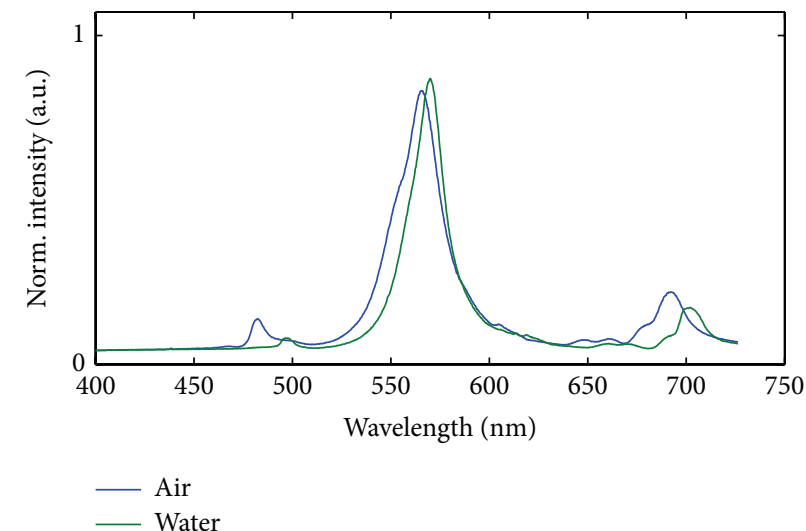

(b) C3b: $t_{1}=0.5\left|t_{2}=0.25\right| t_{3}=0.2$ (meas.)

Figure 6: (a) Simulated and (b) measured reflectance spectra of the compound multiperiodic nanostructure C3b $\left(t_{1}=0.5\left|t_{2}=0.25\right| t_{3}=\right.$ 0.2 ). The second and third dominant peaks are not in the experimental range. 


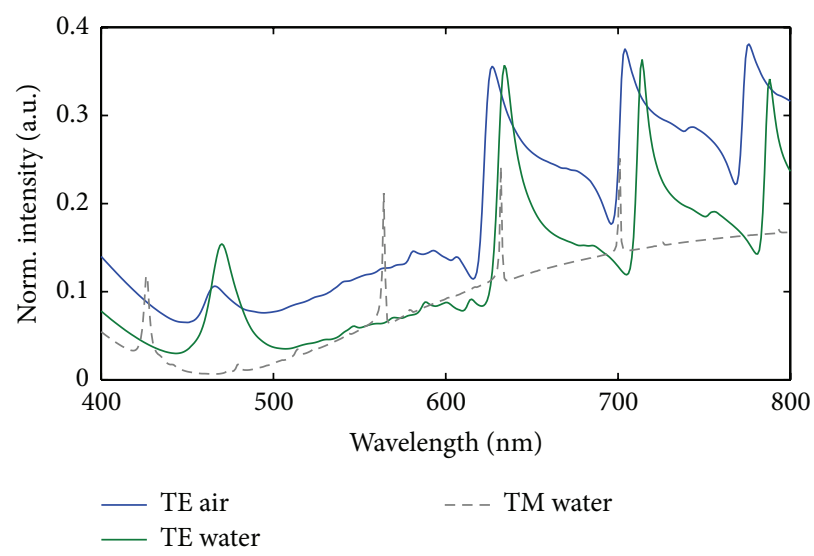

FIGURE 7: Simulated reflectance spectra of the compound multiperiodic nanostructure C4 $\left(t_{1-4}=0.2\right)$. Four dominant resonances are visible.

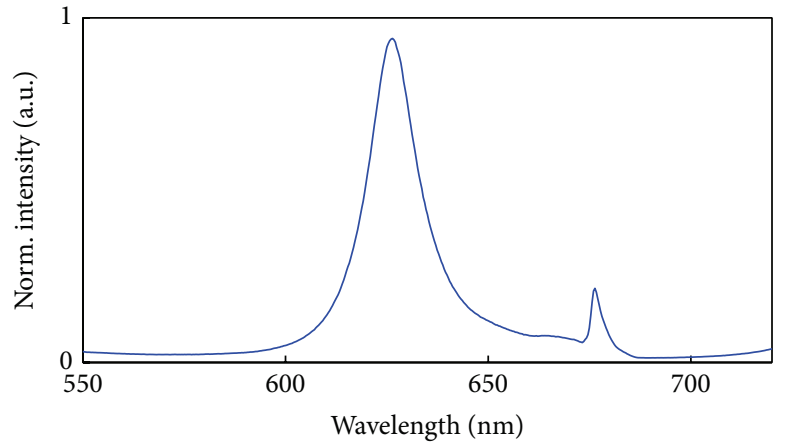

(a) C3a: $t_{1}=0.29\left|t_{2}=0.25\right| t_{3}=0.22$ (meas.)

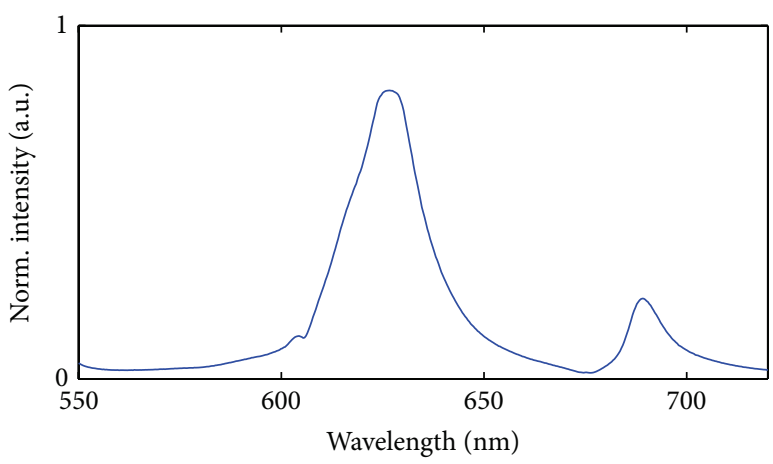

(c) C3b: $t_{1}=0.5\left|t_{2}=0.25\right| t_{3}=0.2$ (meas.)

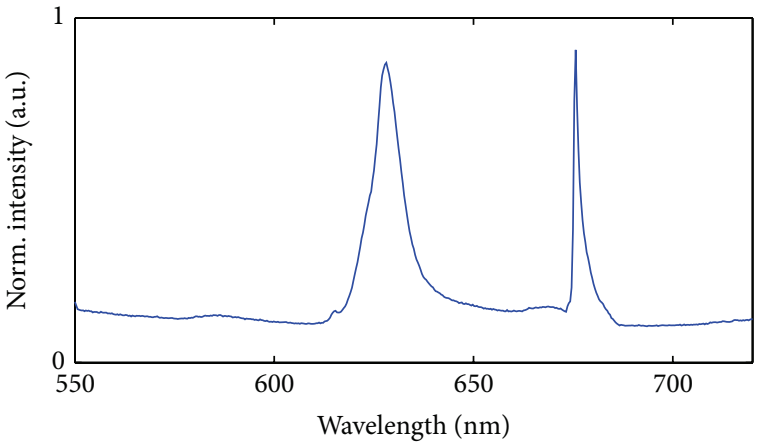

(b) C3a $t_{1}=0.14\left|t_{2}=0.25\right| t_{3}=0.22$ (meas.)

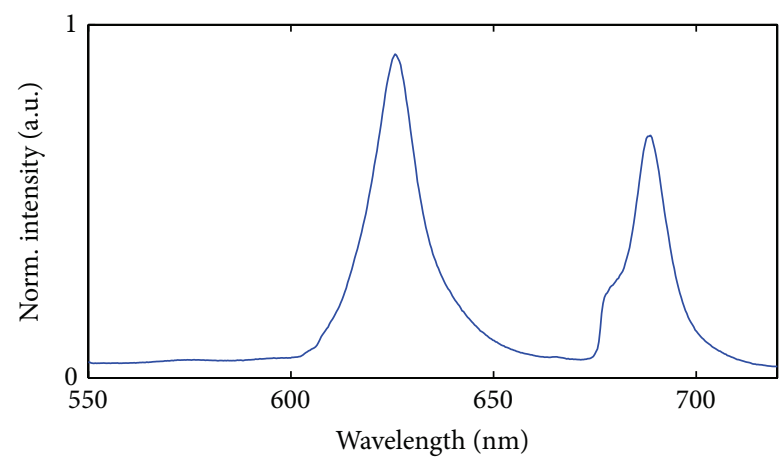

(d) C3b: $t_{1}=0.33\left|t_{2}=0.25\right| t_{3}=0.2$ (meas.)

FIGURE 8: Comparison of measured spectra with air as analyte for structures with varying duty cycle ratios. For these measurements we used a slightly thinner waveguide to investigate the resonances in the visible light regime.

of $S=50 \mathrm{~nm} / \mathrm{RIU} \pm 1.9 \mathrm{~nm} / \mathrm{RIU}$ is observed experimentally for the TM resonance at $550 \mathrm{~nm}$ for the three-period compound nanostructure C3a.

The simulated and measured Q-factors for selected resonances are listed in Table 3. A tendency toward an increase of the Q-factor with the number of grating periods in the compound grating is observed in the simulation. This is contrary to the experimental results. We attribute this difference to the difference in simulated and experimental nanostructure geometries as seen in the comparison of Figures 1 and 2(a). In order to obtain a closer agreement between experimental and simulation results a modified simulation approach needs to be implemented to correctly predict Qfactors. Therefore, the simulation values presented here are valid for the nanostructure geometry shown in Figure 1. As our experimental results show they cannot be applied directly to modified geometries.

3.3. Aperiodic Nanostructures. After comparison of the compound multiperiodic gratings with each other and to a monoperiodic grating, here we compare the compound periodic gratings to aperiodic nanostructures. Simulated reflection spectra for the Rudin-Shapiro (RS), Thue-Morse (ThM), and Fibonacci (F) nanostructures are given in Figure 8. The 
TABLE 2: Sensitivities $S$ of the dominant resonance peaks $\lambda_{R}$ for monoperiodic and multiperiodic nanostructures defined in Table 1 as calculated from the spectra in Figures 4-7. Measurement values are averaged values of the results from eight different nanostructures with different duty cycles but the same periodicities as specified in Table 1. The standard deviation $\sigma$ is listed for the measurement values.

(a) TE polarization

\begin{tabular}{lcccc}
\hline Structure & $\lambda_{R}[\mathrm{~nm}]$ & $\begin{array}{c}S(\mathrm{sim} .) \\
{[\mathrm{nm} / \mathrm{RIU}]}\end{array}$ & $\begin{array}{c}S \text { (meas.) } \\
{[\mathrm{nm} / \mathrm{RIU}]}\end{array}$ & $\begin{array}{c}\sigma(S) \\
(\text { meas. })\end{array}$ \\
\hline $\mathrm{M}$ & 700 & 33 & 32 & - \\
\hline $\mathrm{C} 2$ & 600 & 15 & 22 & 1.3 \\
& 770 & 38 & - & - \\
\hline \multirow{3}{*}{$\mathrm{C} 3 \mathrm{a}$} & 610 & 23 & 24 & 2.9 \\
& 700 & 31 & 31 & 1.2 \\
& 770 & 37 & - & - \\
\hline \multirow{3}{*}{ C3b } & 550 & 12 & 13 & 1.4 \\
& 700 & 30 & 30 & 1.0 \\
& 800 & 41 & - & - \\
\hline \multirow{3}{*}{$\mathrm{C} 4$} & 580 & 12 & - & - \\
& 610 & 22 & - & - \\
& 710 & 24 & - & - \\
\hline
\end{tabular}

\begin{tabular}{lcccc}
\multicolumn{5}{c}{ (b) TM polarization } \\
\hline Structure & $\lambda_{R}[\mathrm{~nm}]$ & $\begin{array}{c}S(\text { sim. }) \\
{[\mathrm{nm} / \mathrm{RIU}]}\end{array}$ & $\begin{array}{c}S \text { (meas. }) \\
{[\mathrm{nm} / \mathrm{RIU}]}\end{array}$ & $\begin{array}{c}\sigma(S) \\
(\text { meas. })\end{array}$ \\
\hline C2 & 550 & 25 & 42 & 8.7 \\
\hline C3a & 550 & 23 & 50 & 1.9 \\
& 600 & 31 & 28 & 2.3 \\
\hline \multirow{3}{*}{ C3b } & 485 & 44 & 43 & 2.6 \\
& 600 & 47 & 44 & 1.0 \\
& 760 & 27 & - & - \\
\hline \multirow{4}{*}{ C4 } & 410 & 22 & - & - \\
& 550 & 46 & - & - \\
& 640 & 49 & - & - \\
\hline
\end{tabular}

existence of guided mode resonances is visible. The intensity of the resonance peaks is even lower than for the compound multiperiodic gratings and substantially lower than for the monoperiodic structure. Compared to compound multiperiodic nanostructures, the aperiodic structures exhibit a higher number of resonance peaks. The most disordered nanostructure, the RS structures, exhibits the most resonance peaks, with the ThM structure following. The Fibonacci structure shows fewer resonances with three strong resonances in a small spectral range.

The sensitivities for selected resonance peaks are calculated and summarized in Table 4. As for the compound multiperiodic gratings, the sensitivities of the TE resonances are overall somewhat lower than for the TM resonances.
TABLE 3: Simulated and measured $Q$-factors for selected resonances of compound multiperiodic gratings (see Figures 4-7) and the reference monoperiodic grating $\mathrm{M}$.

\begin{tabular}{lccc}
\hline Structure & $\lambda_{R}[\mathrm{~nm}]$ & $Q$ (sim.) & $Q$ (meas.) \\
\hline $\mathrm{M}$ & 700 & 19 & 35 \\
\hline \multirow{2}{*}{ 2 } & 600 & 16 & 32 \\
& 770 & 58 & - \\
\hline \multirow{3}{*}{ C3a } & 610 & 27 & 13 \\
& 700 & 57 & 30 \\
& 770 & 77 & - \\
\hline \multirow{2}{*}{ C3b } & 550 & 24 & 9 \\
& 700 & 68 & 26 \\
\hline \multirow{2}{*}{ C4 } & 800 & 67 & - \\
\hline
\end{tabular}

TABLE 4: Simulated sensitivities for selected resonances of the reflection spectra for aperiodic structures in Figure 9.

(a) TE polarization

\begin{tabular}{lcc}
\hline Structure & $\lambda_{R}[\mathrm{~nm}]$ & $S(\operatorname{sim}).[\mathrm{nm} / \mathrm{RIU}]$ \\
\hline \multirow{3}{*}{ RS } & 450 & 16 \\
& 492 & 19 \\
& 533 & 25 \\
& 595 & 26 \\
& 740 & 49 \\
\hline \multirow{2}{*}{ ThM } & 418 & 19 \\
& 530 & 18 \\
& 544 & 20 \\
F & 657 & 33 \\
\hline
\end{tabular}

(b) TM polarization

\begin{tabular}{lcc}
\hline Structure & $\lambda_{R}[\mathrm{~nm}]$ & $S(\operatorname{sim}).[\mathrm{nm} / \mathrm{RIU}]$ \\
\hline \multirow{4}{*}{ RS } & 446 & 18 \\
& 470 & 45 \\
& 490 & 45 \\
& 568 & 45 \\
& 601 & 34 \\
\multirow{2}{*}{ ThM } & 456 & 24 \\
& 471 & 44 \\
& 514 & 44 \\
F & 527 & 40 \\
\hline
\end{tabular}

This is in good agreement with theoretical results for twodimensional structures [29]. Interestingly, in the TM case the sensitivity seems to be mostly independent of the wavelength rather than in the monoperiodic case, where higher wavelengths and higher sensitivity are correlated. Overall similar 


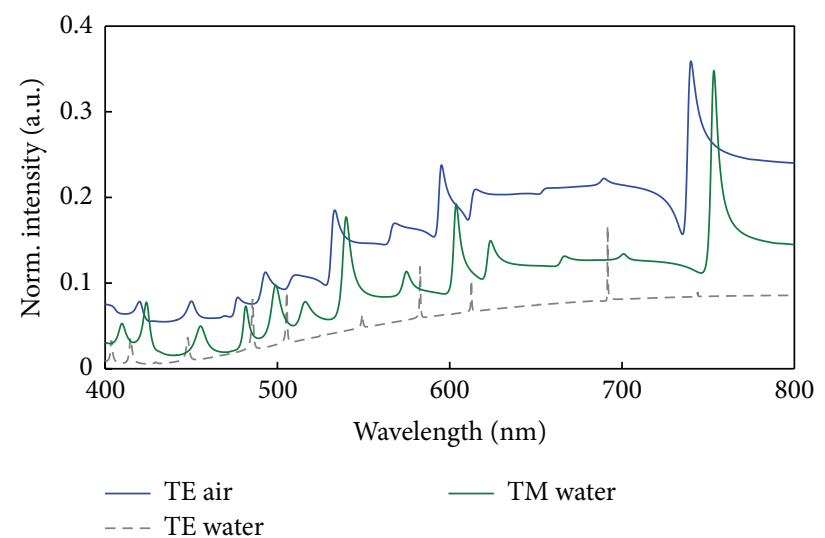

(a) Rudin-Shapiro (sim.)

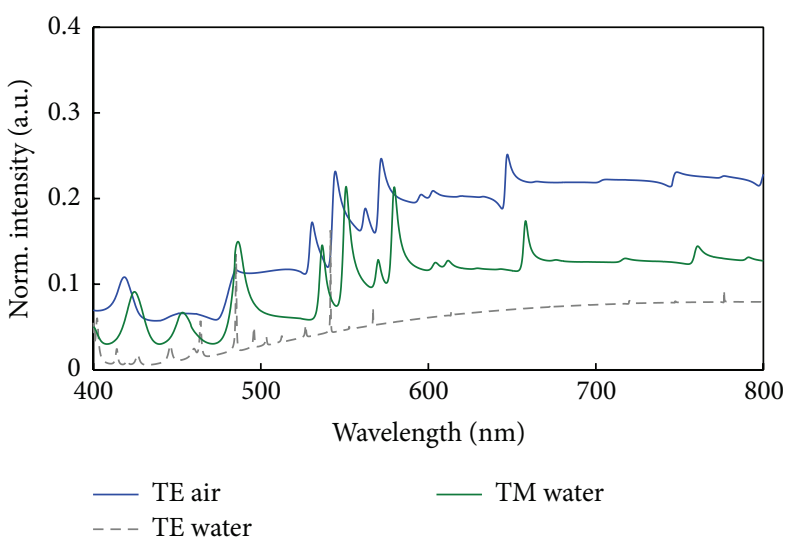

(b) Thue-Morse (sim.)

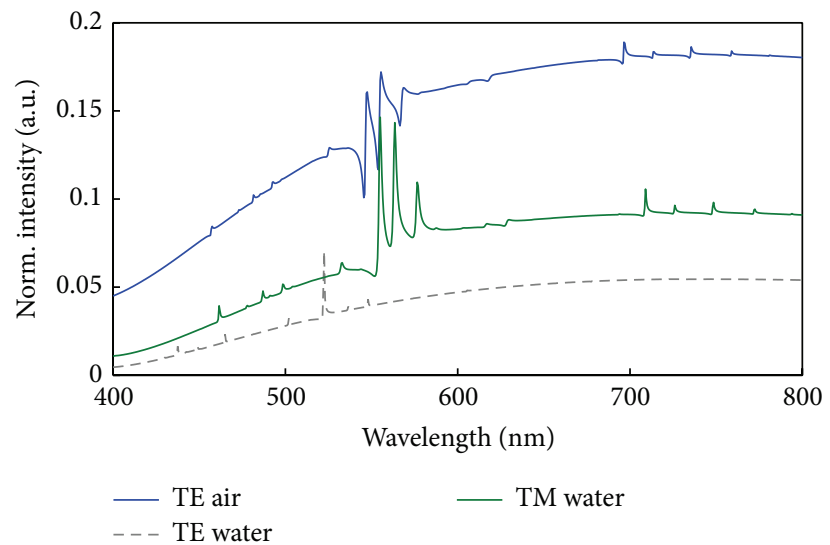

(c) Fibonacci (sim.)

FIGURE 9: Simulated reflection spectra for the Rudin-Shapiro, Thue-Morse, and Fibonacci-nanostructures.

TABLE 5: Q-factors for various resonances of the RS structure. The $Q$-factors for more complex structures are greatly enhanced.

\begin{tabular}{lcc}
\hline Structure & $\lambda_{R}[\mathrm{~nm}]$ & $Q$ (sim.) \\
\hline \multirow{4}{*}{ RS } & 455 & 62 \\
& 499 & 73 \\
& 540 & 95 \\
& 604 & 104 \\
& 753 & 150 \\
\hline
\end{tabular}

sensitivities are observed for aperiodic gratings and compound multiperiodic gratings.

Finally, we calculated the $Q$-factors of the resonance peaks in the reflection spectra of the RS structure, as given in Table 5. The spectrum exhibits resonances with highly increased Q-factors in comparison to the compound multiperiodic gratings.

\section{Conclusion}

We designed, fabricated, and characterized 24 different compound multiperiodic nanostructured waveguides for refractive index sensing. The results are compared to a monoperiodic nanostructure as well as to three selected aperiodic nanostructures (Rudin-Shapiro, Fibonacci, and Thue-Morse). All structures have the same waveguide geometry such that only the nanostructure design is changed. Considering (1) we expect a similar sensitivity for all nanostructures as long as the nanostructure does not change the local electric field distribution significantly. Figure 10 summarizes all obtained sensitivity values as a function of wavelength and polarization. The sensitivity of TE resonances increases with wavelength. This increased sensitivity may be attributed to the larger overlap of the mode with the analyte at higher wavelengths. The sensitivity of the TE resonances is similar for all types of compound multiperiodic nanostructures, while higher sensitivity values are observed for aperiodic nanostructures. This indicates that the local electric field distribution is changed for the aperiodic nanostructures allowing for a higher sensitivity. In general, higher sensitivity values are achieved for TM resonances. For the TM resonances the compound multiperiodic nanostructures exhibit higher sensitivity values compared to the monoperiodic nanostructure and similar values as the aperiodic nanostructures. The sensitivity of the TM resonances does not show a clear correlation with the resonance wavelength. For a more detailed investigation of these effects, the field distributions in the nanostructures should be compared in future work. This study indicates the influence of the local field distribution on the sensitivity. 


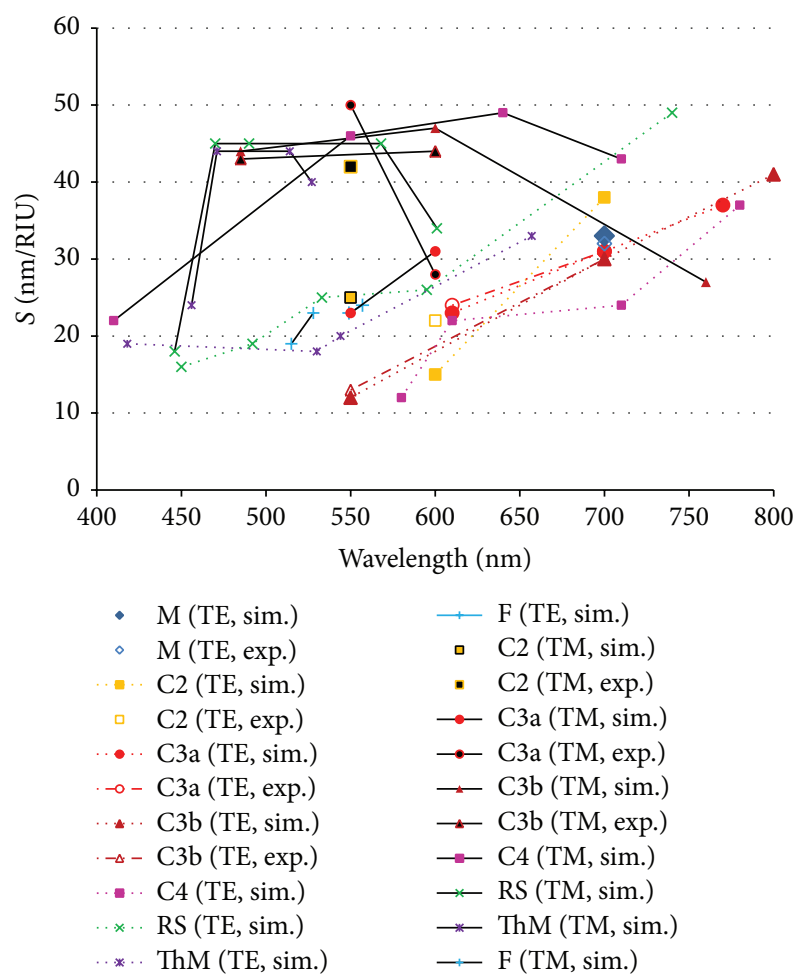

FIGURE 10: Sensitivities of all investigated nanostructures as a function of wavelength. Structure details for monoperiodic (M), compound multiperiodic (C), Rudin-Shapiro (RS), Thue-Morse (ThM), and Fibonacci (F) nanostructures are given in Table 1. Obtained simulation (sim.) and experimental (exp.) results are summarized for TE polarization and TM polarization.

Therefore, the nanostructure cannot be seen as a simple coupling structure but significantly influences the sensitivity. No significant influence of the compound grating duty cycles on the sensitivity is observed as the standard deviations in Table 2 show. The duty cycles allow for adjusting the relative peak heights.

Compared to monoperiodic and aperiodic nanostructures lower Q-factors are observed for compound multiperiodic nanostructures. Such lower $Q$-factor corresponds to a shorter scattering length and thus a shorter photon lifetime in the nanostructure [8]. This indicates that compound multiperiodic nanostructures scatter photons more efficiently. We expect that the $Q$-factor of all the investigated structures may be tuned by varying the refractive index contrast and the grating depth. Ding and Magnusson [9] have demonstrated that these two factors define the modulation strength and allow for a tailoring of the resonance width. In the scope of this study, we compared different nanostructures with identical modulation strength. For applications requiring high sensitivity and high $Q$-factor, the modulation strength should be adapted.

In conclusion, compound multiperiodic nanostructures offer the unique advantage that wavelengths and number and intensities of resonances can be tailored. This allows for new sensor system approaches based on designing a specific spectral fingerprint for unambiguous and reliable refractive index sensing. Due to the lower Q-factor the compound multiperiodic nanostructures in this investigation seem particularly promising for spectrometer-free sensor systems evaluating the integrated resonance intensity in different wavelength bands. Here, a moderate $Q$-factor combined with a high sensitivity is desirable.

\section{Conflict of Interests}

The authors declare no conflict of interests.

\section{Acknowledgments}

The authors acknowledge support by the European Research Council within the project Photosmart (307800). This work was carried out with the support of the Karlsruhe Nano and Micro Facility (KNMF, http://www.kit.edu/knmf), a Helmholtz Research Infrastructure at Karlsruhe Institute of Technology (KIT, http://www.kit.edu/).

\section{References}

[1] R. Magnusson, D. Wawro, S. Zimmerman, and Y. Ding, "Resonant photonic biosensors with polarization-based multiparametric discrimination in each channel," Sensors, vol. 11, no. 2, pp. 1476-1488, 2011.

[2] Y. Fang, "Label-free cell-based assays with optical biosensors in drug discovery," Assay and Drug Development Technologies, vol. 4, no. 5, pp. 583-595, 2006.

[3] B. Cunningham, M. Zhang, Y. Zhuo, L. Kwon, and C. Race, "Review of recent advances in biosensing with photonic crystal surfaces," IEEE Sensors Journal, 2015.

[4] D. Threm, Y. Nazirizadeh, and M. Gerken, "Photonic crystal biosensors towards on-chip integration," Journal of Biophotonics, vol. 5, no. 8-9, pp. 601-616, 2012.

[5] I. M. White and X. Fan, "On the performance quantification of resonant refractive index sensors," Optics Express, vol. 16, no. 2, pp. 1020-1028, 2008.

[6] F. Vollmer, L. Yang, and S. Fainman, "Label-free detection with high-Q microcavities: a review of biosensing mechanisms for integrated devices," Nanophotonics, vol. 1, no. 3-4, pp. 267-291, 2012.

[7] Z. Yu and S. Fan, "Extraordinarily high spectral sensitivity in refractive index sensors using multiple optical modes," Optics Express, vol. 19, no. 11, pp. 10029-10040, 2011.

[8] I. D. Block, P. C. Mathias, S. I. Jones, L. O. Vodkin, and B. T. Cunningham, "Optimizing the spatial resolution of photonic crystal label-free imaging," Applied Optics, vol. 48, no. 34, pp. 6567-6574, 2009.

[9] Y. Ding and R. Magnusson, "Resonant leaky-mode spectralband engineering and device applications," Optics Express, vol. 12, no. 23, pp. 5661-5674, 2004.

[10] C. Nicolaou, W. T. Lau, R. Gad, H. Akhavan, R. Schilling, and O. Levi, "Enhanced detection limit by dark mode perturbation in 2D photonic crystal slab refractive index sensors," Optics Express, vol. 21, no. 25, pp. 31698-31712, 2013.

[11] S. V. Boriskina and L. Dal Negro, "Sensitive label-free biosensing using critical modes in aperiodic photonic structures," Optics Express, vol. 16, no. 17, pp. 12511-12522, 2008. 
[12] L. Dal Negro and S. V. Boriskina, "Deterministic aperiodic nanostructures for photonics and plasmonics applications," Laser and Photonics Reviews, vol. 6, no. 2, pp. 178-218, 2012.

[13] L. Dal Negro, "Enhancing optical biosensing with aperiodicity," SPIE Newsroom, 2012.

[14] E. MacIá, "Exploiting aperiodic designs in nanophotonic devices," Reports on Progress in Physics, vol. 75, no. 3, Article ID 036502, 2012.

[15] Z. V. Vardeny, A. Nahata, and A. Agrawal, "Optics of photonic quasicrystals," Nature Photonics, vol. 7, no. 3, pp. 177-187, 2013.

[16] M. Renner and G. von Freymann, "Transverse mode localization in three-dimensional deterministic aperiodic structures," Advanced Optical Materials, vol. 2, no. 3, pp. 226-230, 2014.

[17] C. Kluge, J. Adam, N. Barié, P.-J. Jakobs, M. Guttmann, and M. Gerken, "Multi-periodic nanostructures for photon control," Optics Express, vol. 22, no. 5, pp. A1363-A1371, 2014.

[18] C. Kluge, L. T. Neustock, J. Adam, and M. Gerken, "Wavelength dependency of outcoupling peak intensities for emission layers with multi-periodic photonic crystals," in Proceedings of the 16th International Conference on Transparent Optical Networks (ICTON '14), pp. 1-4, Graz, Austria, July 2014.

[19] W. Steurer and D. Sutter-Widmer, "Photonic and phononic quasicrystals," Journal of Physics D: Applied Physics, vol. 40, no. 13, pp. R229-R247, 2007.

[20] S. Y. Boriskina, A. Gopinath, and L. Dal Negro, "Optical gap formation and localization properties of optical modes in deterministic aperiodic photonic structures," Optics Express, vol. 16, no. 23, pp. 18813-18826, 2008.

[21] M. G. Moharam, E. B. Grann, D. A. Pommet, and T. K. Gaylor, "Formulation for stable and efficient implementation of the rigorous coupled-wave analysis of binary gratings," Journal of the Optical Society of America A, vol. 12, no. 5, pp. 1068-1076, 1995.

[22] Y. Ding and R. Magnusson, "Band gaps and leaky-wave effects in resonant photonic-crystal waveguides," Optics Express, vol. 15, no. 2, pp. 680-694, 2007.

[23] P. Lalanne and E. Silberstein, "Fourier-modal methods applied to waveguide computational problems," Optics Letters, vol. 25, no. 15, pp. 1092-1094, 2000.

[24] A. Ricciardi, I. Gallina, S. Campopiano et al., "Guided resonances in photonic quasicrystals," Optics Express, vol. 17, no. 8, pp. 6335-6346, 2009.

[25] S. Jahns, F. Von Oertzen, T. Karrock, Y. Nazirizadeh, and M. Gerken, "Photonic crystal slabs for biosensing," in Proceedings of the Progress in Electromagnetics Research Symposium (PIERS '14), pp. 1217-1220, Guangzhou, China, August 2014.

[26] Y. Nazirizadeh, U. Bog, S. Sekula, T. Mappes, U. Lemmer, and M. Gerken, "Low-cost label-free biosensors using photonic crystals embedded between crossed polarizers," Optics Express, vol. 18, no. 18, pp. 19120-19128, 2010.

[27] S. G. Johnson, S. Fan, P. R. Villeneuve, J. D. Joannopoulos, and L. A. Kolodziejski, "Guided modes in photonic crystal slabs," Physical Review B, vol. 60, no. 8, pp. 5751-5758, 1999.

[28] D. Rosenblatt, A. Sharon, and A. A. Friesem, "Resonant grating waveguide structures," IEEE Journal of Quantum Electronics, vol. 33, no. 11, pp. 2038-2059, 1997.

[29] M. Pisco, A. Ricciardi, I. Gallina et al., "Tuning efficiency and sensitivity of guided resonances in photonic crystals and quasicrystals: a comparative study," Optics Express, vol. 18, no. 16, pp. 17280-17293, 2010. 


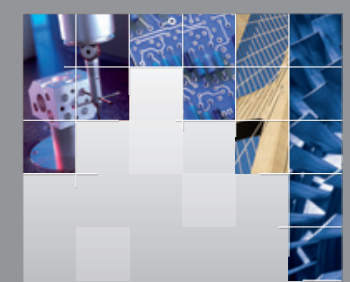

\section{Enfincering}
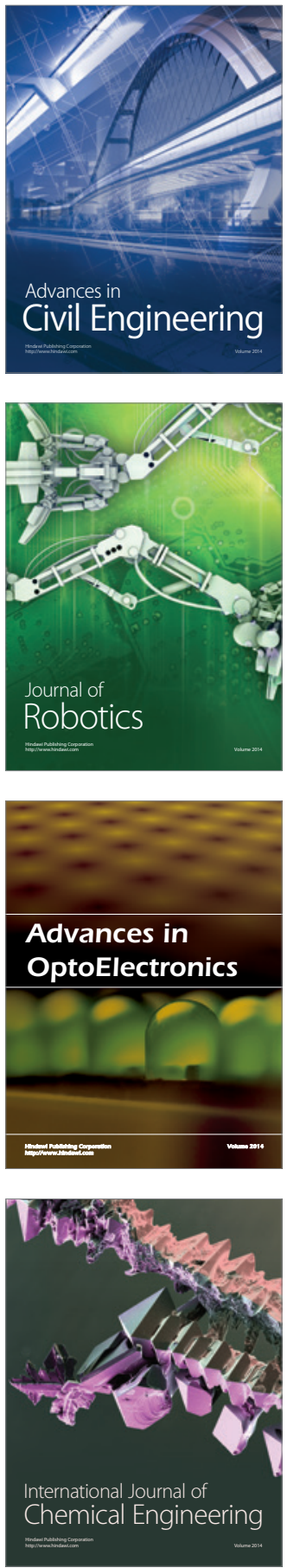



The Scientific World Journal

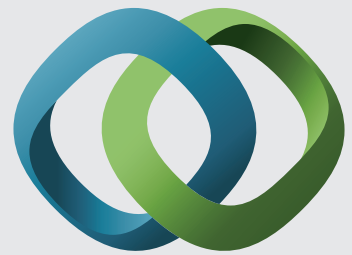

\section{Hindawi}

Submit your manuscripts at

http://www.hindawi.com
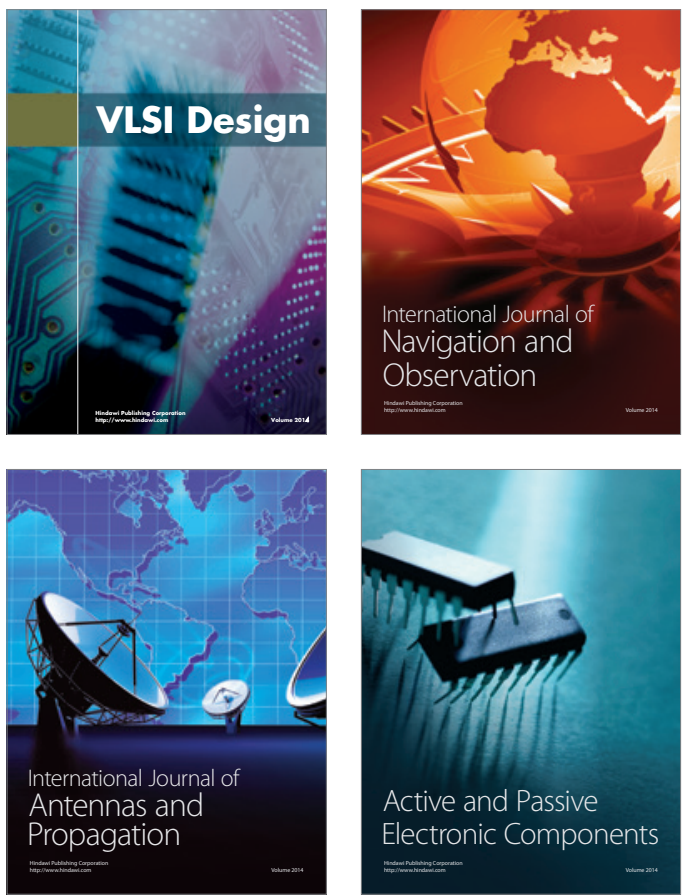
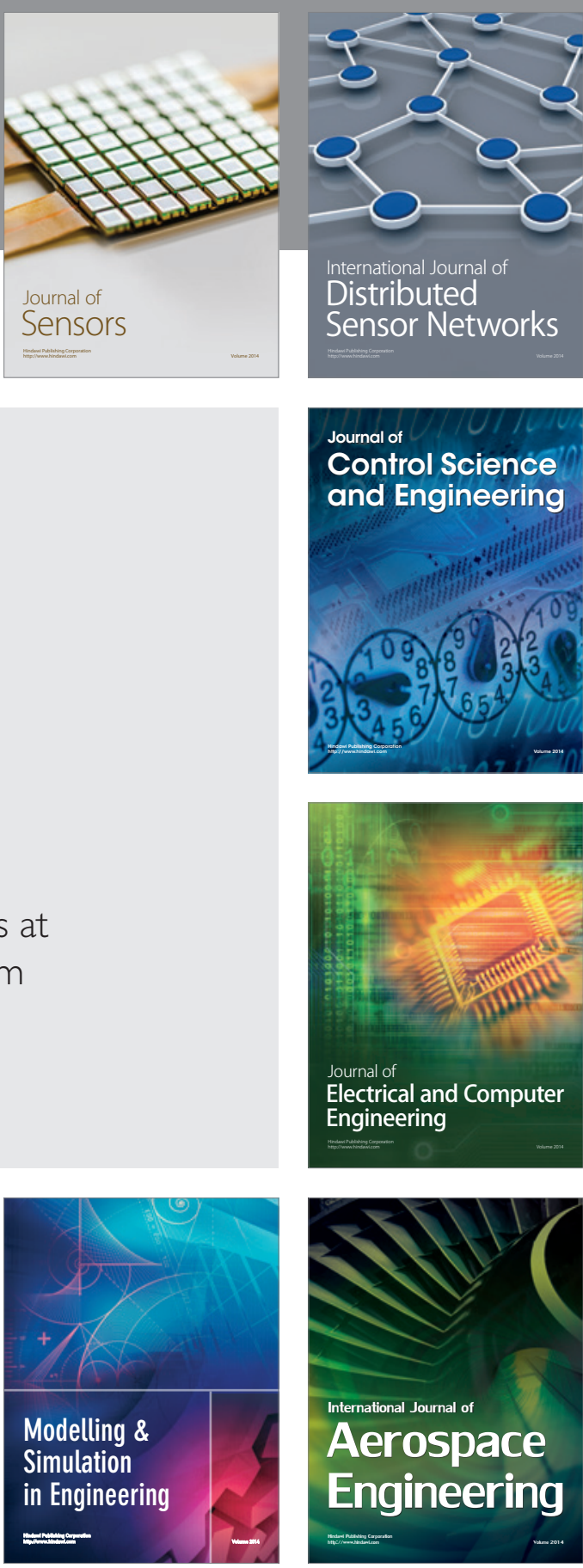

International Journal of

Distributed

Sensor Networks

Journal of

Control Science

and Engineering
